\title{
INDOOR EVALUATION OF CROP ROW AND GRID DETECTION System for an automated transplanter
}

\author{
J. Reumers ${ }^{1}$, F. De Smedt ${ }^{2}$, J. Anthonis ${ }^{1}$, H. Ramon ${ }^{1}$ and T. Goedemé ${ }^{2}$ \\ ${ }^{1}$ Department for Agro-Engineering and Economics, K.U. Leuven, Kasteelpark 30, 3001 Leuven, Belgium \\ ${ }^{2}$ EAVISE, Lessius Mechelen, Association K.U.Leuven, De Nayerlaan 5, 2860 Sint-Katelijne-Waver, Belgium \\ Jan.Anthonis@agr.kuleuven.be
}

Keywords: agriculture, crop detection, vehicle guidance, transplanter

\begin{abstract}
If crops with considerable spacing can be arranged in a precise rectangular pattern, mechanical weeding can become an alternative to chemical methods by enabling treatment in two perpendicular directions. Realisation of such a pattern requires innovation of seedling transplanters. A computer vision-based sensing system was developed for detecting a transplanters posture relative to the crop. A method for indoor evaluation of the systems accuracy is proposed, using an experimental cart on a set of rails to control the vehicle's posture. The method was successful in evaluating the estimates of the lateral offset and the heading angle, but the reliability of the validation values for the longitudinal distance is limited. Tests results showed that the maximum error on the measurements of the lateral offset is $1.5 \mathrm{~cm}$ and that the standard deviation is smaller than $0.6 \mathrm{~cm}$. The maximum error on the angle measurements is $2.1^{\circ}$. The standard deviation of the error is smaller than $0.65^{\circ}$. The standard deviation of the error on the estimates of the longitudinal distance is typically $0.7 \mathrm{~cm}$.
\end{abstract}

\section{INTRODUCTION}

An integrated approach could promote mechanical weed control to become an efficient, adequate alternative to traditional chemical treatment in crops with considerable spacing. If the crop can be arranged in a regular rectangular planting pattern, the applicability, working rate and effectiveness of interrow weeding increases and mechanical treatment becomes possible in a second, perpendicular direction. This would circumvent the problem of weed removal in the rows and would considerably increase the percentage area treated (Schoenfish and Billingsley, 1993),(Søgaard and Olsen, 2003). Arrangement of the crop according to a precise rectangular grid is currently not possible. In (Reumers et al., 2005a) a vision-based sensing system for an automated seedling transplanter was presented. This crop detection system provides the posture information (two-dimensional position and orientation relative to the crop) required to control the transplanter for realisation of regular grid pattern. It might also be applied in early growth stages for guidance of mechanical weeders to improve the quality and working rate of the treatment. Automation of transplanting and weeding will improve operator comfort as well, by relieving him from the repetitive and tiring low-level tasks, enabling him to concentrate on supervising functions, multitasking, the working performance of the implement and machine safety. Plant specific treatment in early stages of crop development is another possible application domain (Tillet et al., 2002).

The computer vision system measures three parameters that define the posture of the vehicle or implement: the heading angle relative to the crop rows, the lateral offset $\mathrm{O}$ relative to the crop row next to the vehicle and the distance $\mathrm{N}$ along the driving direction to the next plant position. (Reumers et al., 2005b) conducted outdoor trials of the system. It was capable of detecting and tracking the crop grid under real field conditions with natural illumination, but the accuracy of measurements could be evaluated only for the lateral offset. In this paper an indoor evaluation procedure is presented and test results are analysed.

\section{METHODOLOGY}

\subsection{Vision system hardware}

A commercially available RGB/CIR camera was selected (DuncanTech MS-2150). This 3 CCD camera acquires images consisting of 4 colour planes: NIR 
$(768-832 \mathrm{~nm})$, red $(640-680 \mathrm{~nm})$, green $(520-560 \mathrm{~nm})$ and blue $(438-482 \mathrm{~nm})$.

A spatial calibration method was reported in (Reumers et al., 2005b) is used for the construction of a transformation table for fast conversion of image coordinates to real world coordinates. The displacement between successive images is used to accelerate the image processing algorithms and to provide more robustness to illumination variations and to weeds.

\subsection{Crop detection program}

The crop detection system we propose is robust to a wide range of natural conditions, highly variable in intensity and spectral content, both over time and space. A large range of soil and crop types (both green and non-green) can be dealt with. Soil coverage by the seedlings is very small upon transplanting, sometimes less than $1 \%$. In horicultural applications, the space between plants can be large, which forms an extra objective (Southall, 1999). The image analysis algorithms are developed specifically to exploit the contextual information of this situation.

The main features of the image-processing loop are segmentation, line or grid fitting and determination of search region positions. The segmentation is based on the normalised difference vegetation index (De Smedt et al., 2011). This technique combines the red and near-infrared information. Over monochrome NIR images, the use of the NDVI offers the advantage of much higher robustness to changes in illumination intensity. The use of automatic thresholding is suffering from the low percentage of soil coverage by the crop. Therefor at program initiation, a fixed empirical threshold level is used. Once sufficient plants are detected, search regions are constructed. The increased soil coverage inside the search regions allows the use of automatic thresholding. This unique approach introduces exceptional robustness to changes in illumination intensity and even spectral content.

The plants within the field of view should not touch or overlap each other and the size of the weeds close to the crop must be smaller than the size of the crop for a correct segmentation. Upon transplanting of seedlings and during early crop development stages these conditions are met.

Plant positions in a new image are predicted based on their previous position and vehicle displacement. The predicted positions determine the search regions locations. New search regions are defined at the location where new plants will enter the side of view. This approach prevents the need to process the entire image and analyse only the relevant information.

In the binary image resulting from the segmenta- tion step, individual plants are identified based on size and position. The plant centres are chosen to represent plant positions. The detected plant positions are used to fit lines marking crop row positions. Both a line fit method and a grid fit method are implemented, based on least squares to minimise the distance between the fitted line/grid and the plant positions.

The line method fits a line for each row separately and then integrates this information by the calculation of parameter averages, weighed according to the number of plants in each row. The equations of the fitted lines allow the calculation of the desired output values $\mathrm{O}, \theta$ and $\mathrm{N}$. The grid fit technique increases robustness and accuracy by integrating all two-dimensional information of plant positions in a single optimisation. Still it is possible to perform a trade-off between the accuracy importance of the longitudinal rows and the accuracy importance of the lateral rows. The grid fit method needs to be initialised by a previous grid position estimate, which can be provided by the line fit method. Note that both fit methods are performed in the real world coordinate system. Some of the output signals show high frequency noise, probably due to camera vibrations and the nature of the application. This can be solved by the use of a low-pass filter.

\subsection{Indoor evaluation tests}

For our experiments, we use a setup of two rails (18m) on which an experimental vehicle is able to ride. In between these rails soil was spread and two rows of lettuce were planted (32 plants), according to a rectangular grid. This setup allows a very accurate validation of the measurement data. Two different camera mountings where applied on the vehicle (figure 1).

During a test the vehicle was pushed manually in order to move the vision system along the crop rows. All experiments were conducted at four different driving speeds named slow (0.4 to $0.5 \mathrm{~m} / \mathrm{s})$, medium $(0.7$ to $1.0 \mathrm{~m} / \mathrm{s})$, fast ( 1.3 to $1.6 \mathrm{~m} / \mathrm{s})$ and very fast $(2.1$ to $2.3 \mathrm{~m} / \mathrm{s})$. To prevent motion blur, six halogen lights $(500 \mathrm{~W})$ where used to decrease integration time. These are mounted on the vehicle.

To obtain validation data, the following procedures were followed. Since the orientation of the camera relative to the rails, and hence relative to the crop rows, is constant during a test, the validation value of the heading angle $\theta$ is constant for a certain camera mounting as well. Moreover, both the crop rows and the calibration pattern are placed parallel to the rails. Consequently the validation value of the heading angle $\theta$ is equal to 0 , as during spatial calibration the coordinate system is defined parallel to the pattern.

Note that the camera need not be aligned with the 


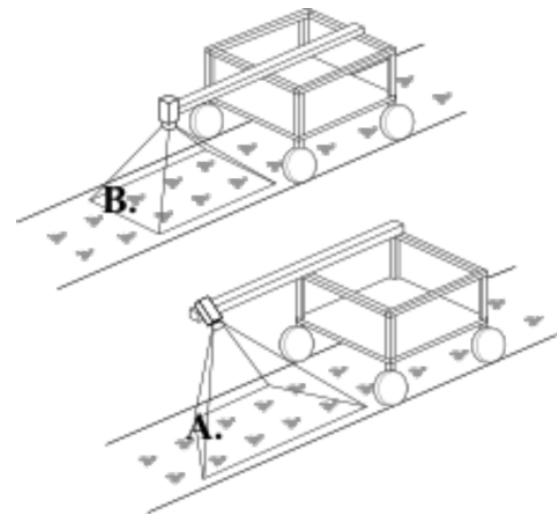

Figure 1: Camera mountings: A. Vertical, B. Sidewards

rails. This is a big advantage, since perfect alignment is hard to achieve. The point of interest for which the posture estimates are calculated was chosen to be the centre of the field of view (the point of projection of the optical axis of the camera). The validation value of the lateral offset $\mathrm{O}$ of the camera is a constant for a certain mounting as well. It is the distance between the centre of the field of view and the longitudinal reference crop row. This distance is equal to the difference between the measured distances of the centre of the field of view and the reference row, both relative to the left rail. As the validation values for $\mathrm{O}$ and $\theta$ are constants, problems with linking or synchronisation of measurements and validation data are not an issue. For validation purposes, the longitudinal position of the vehicle is measured using an optical sensor. This sensor measures the distance from the starting point based a strip of paper on the floor with alternating black and white stripes. The accuracy of the position measurement is $1 \mathrm{~cm}$. The system proved to be very reliable.

Relative to the same starting point, the longitudinal positions of the plants were measured manually, using the strip with black and white stripes as a ruler. For evaluation, each of the distances $\mathrm{N}$ to the next lateral row computed by the crop detection program was logged together with corresponding counter values. These counter values are read prior to locking the most recent frame for processing. During a validation test, the vehicle is driven across the rails. Starting from the first black line on the paper strip, the counter starts monitoring the longitudinal position of the vehicle. For each processed image the longitudinal position measured by the optical sensor and the posture parameters calculated by the vision system are saved to a $\log$ file. Validation can be done by comparing the calculated and measured values.

\section{Results and discussion}

The accuracies of $\mathrm{O}, \mathrm{N}$ and $\theta$ were analysed for a sideways camera moved at medium speed.Figure 2 shows the results from the comparison of the crop detection estimate to the validation data.

Figure 3 compares the estimated distances to the next lateral row $\mathrm{N}$ to their corresponding validation values. The estimates are shifted to the right due to a potential systematic error of the system and the time delay between acquisition of the validation data and the corresponding images.

The statistics of the errors on estimates of $\mathrm{O}$ and $\theta$ are given in table 1 and table 3 . We will present the results for filtered values obtained by the grid fit method, since these gives the best results. The given results for $\mathrm{N}$ are taken on unfiltered data.

Table 1 present the statistics of the filtered offset estimates. Both the standard deviation and the maximum error are considerably smaller than those of the unfiltered data. The systematic deviations remain generally unchanged. There is no clear influence of camera mounting.

The accuracy of the angle measurements can be judged with the help of table 3 . We can observe that the influence of the driving speed is minimal. Sidewards camera mounting is slightly better than vertical mounting using the grid fit method. Table 2 summarise the results of the distance estimates $\mathrm{N}$ to the next lateral row. The systematic deviations and maximum errors are considerably increasing with increasing driving speed. This is due to the lack of synchronisation of the acquisition of images and the retrieval of counter values. This can lead to a difference up to $10 \mathrm{~cm}$ between the measured position by the optical sensor and the real position. This combined with the absence of a real-time operating system can lead to very big errors. These extreme outliers have been removed for the calculation of the statistics.

As the experimental vehicle was driven manually, speed was never constant during a test. Especially at the highest speed a large part of the posture estimates are generated while accelerating or braking. Consequently, the difference between validation value and real position at the moment of image acquisition is not constant during execution of a test. This increases the standard deviations, as can be seen in table 2 . With proper synchronisation these will be smaller. The systematic deviations will be eliminated for the most part. This can be simulated by compensating the maximum errors for the systematic deviation. This is done by subtracting the average errors from the maximum errors. The compensated maximum errors (table 4) are considerably smaller than the non-compensated 
values.

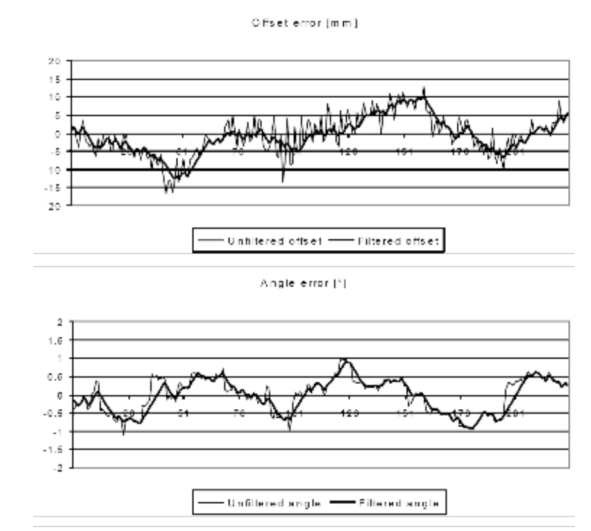

Figure 2: Sample of the course of the unfiltered and filtered offset error (above) and the angle error (below) as a function of the number of images processed (sidewards camera mounting, grid fit method, medium speed)

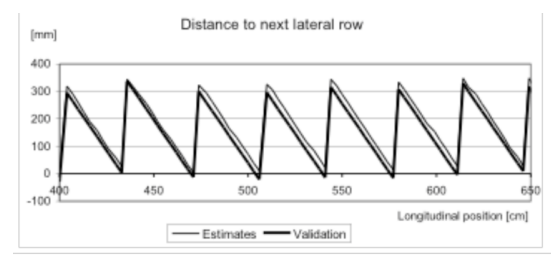

Figure 3: Comparison of the estimated distance to the next lateral row $\mathrm{N}$ and the corresponding validation values as a function of the longitudinal position (sidewards camera mounting,grid fit method, medium speed)

\section{Conclusions}

A vision-based posture system has been developed for the detection of crop row and grid detection, which can be used for many applications. We proposed a line fit method and a grid fit method. We evaluated these on a indoor setup at different speeds. During the evaluation tests the system never lost track of the crop pattern. We observed better results from the use of the grid fit method, while no performance difference is observed. This indicates the higher stability and inherent accuracy of the grid fit method. The (unfiltered) estimates of the longitudinal distance of both methods are equivalent. The influences of camera mounting and vehicle speed on system accuracy were not clear.

The posture measurements by the vision system are sufficiently accurate in general, especially when the grid fit method is applied and the output signals are filtered. The use of a real-time operating system and decent synchronisation might probably reduce the maximum error to less than $2.5 \mathrm{~cm}$ to improve the results even more.

\section{ACKNOWLEDGEMENTS}

This research was funded by the Institute for the Promotion of Innovation through Science and Technology in Flanders (IWT Vlaanderen), N.V. Agriplant and the Interuniversity Attraction Poles Programme Belgian Science Policy. Jan Anthonis is funded as a Post-doctoral Fellow by the Fund for Scientific Research Flanders (Belgium) (FWO Vlaanderen).

\section{REFERENCES}

De Smedt, F., Billiauws, I., and Goedeme, T. (2011). Neural networks and low-cost optical filters for plant segmentation. In International Journal of Computer Information Systems and Industrial Management Applications (IJCISIM), volume 3/4.

Reumers, J., Anthonis, J., Bruyninckx, H., and Ramon, H. (2005a). Detection of crop rows with low soil coverage using computer vision. In Computers and electronics in agriculture.

Reumers, J., Anthonis, J., Van Gool, L., and Ramon, H. (2005b). llumination independent tracking of individual plants with low soil coverage using computer vision. In Computers and electronics in agriculture.

Schoenfish, M. and Billingsley, J. (1993). A comparison of automated guidance systems for a variety of operations in the growing of cotton. In Proceedings international advanced robotics programme $3 r d$ workshop on robotics in agriculture and the food industry, pages $8-16$.

Søgaard, H. and Olsen, H. (2003). Determination of crop rows by image analysis without segmentation. In Computers and electronics in agriculture, volume 38, pages 141-158.

Southall, J. (1999). The development and evaluation of computer vision algorithms for the control of an autonomous horticultural vehicle. $\mathrm{PhD}$ thesis, University of London.

Tillet, N., Hague, T., and Miles, S. (2002). Inter-row vision guidance for mechanical weed control in sugar beet. In Computers and electronics in agriculture, volume 33, pages 163-177. 
Table 1: Offset, grid method, filtered data.

\begin{tabular}{|c|c|c|c|c|c|c|}
\hline Camera mounting & \multicolumn{3}{|c|}{ Vertical } & \multicolumn{3}{c|}{ Sidewards } \\
\hline Driving speed & Average error [mm] & St. Devation [mm] & Max Abs error [mm] & Average error [mm] & St. Deviation [mm] & Max abs. error [mm] \\
\hline Slow & 8.5 & 10.9 & 40.0 & 17.9 & 7.2 & 7.5 \\
\hline Medium & 21.7 & 10.2 & 51.6 & 23.2 & 7.3 \\
\hline Fast & 38.0 & 10.0 & 65.5 & 48.7 & 7.1 \\
\hline Very fast & 58.4 & 14.6 & 95.3 & 72.9 & 1.4 \\
\hline
\end{tabular}

Table 2: Distance to next lateral row, grid fit method

\begin{tabular}{|c|c|c|c|c|c|c|}
\hline Camera mounting & \multicolumn{3}{|c|}{ Vertical } & \multicolumn{2}{c|}{ Sidewards } \\
\hline Driving speed & Average error [mm] & St. Devation [mm] & Max Abs error [mm] & Average error [mm] & St. Deviation [mm] & Max abs. error [mm] \\
\hline Slow & -0.42 & 0.65 & 2.11 & -0.19 & 0.56 & 1.58 \\
\hline Medium & -0.38 & 0.63 & 1.85 & -0.21 & 0.52 \\
\hline Fast & -0.39 & 0.52 & 1.54 & -0.22 & 0.45 \\
\hline Very fast & -0.26 & 0.61 & 1.28 & -0.15 & 1.08 \\
\hline
\end{tabular}

Table 3: Angle, grid fit method, filtered data

\begin{tabular}{|c|c|c|c|c|c|c|}
\hline Camera mounting & \multicolumn{3}{|c|}{ Vertical } & \multicolumn{3}{c|}{ Sidewards } \\
\hline Driving speed & Average error $\left[{ }^{\circ}\right]$ & St. Devation $\left[{ }^{\circ}\right]$ & Max Abs error $\left[{ }^{\circ}\right]$ & Average error $\left[{ }^{\circ}\right]$ & St. Deviation $\left[{ }^{\circ}\right]$ & Max abs. error $\left[{ }^{\circ}\right]$ \\
\hline Slow & -4.3 & 5.3 & 14.4 & -2.8 & 5.8 & 15.2 \\
\hline Medium & -3.5 & 5.0 & 14.8 & -2.4 & 5.5 & 14.6 \\
\hline Fast & -3.2 & 4.4 & 12.8 & -2.5 & 4.6 & 13.0 \\
\hline Very fast & -2.1 & 3.2 & 8.4 & 0.5 & 4.4 & 9.9 \\
\hline
\end{tabular}

Table 4: Compensated maximum errors on the distance to the next lateral row[mm]

\begin{tabular}{|c|c|c|c|c|}
\hline Camera mounting & \multicolumn{2}{|c|}{ Vertical } & \multicolumn{2}{c|}{ Sidewards } \\
\hline Driving speed & Grid fit method & Line fit method & Grid fit method & Line fit method \\
\hline Slow & 31.5 & 31.3 & 24.2 & 25.9 \\
\hline Medium & 3.4 & 34.3 & 24.8 & 20.8 \\
\hline Fast & 27.5 & 31.7 & 20.8 & 18.9 \\
\hline Very fast & 36.9 & 39.8 & 28.7 & 27.4 \\
\hline
\end{tabular}

